\title{
Motion-Compensated MR Valve Imaging with COMB Tag Tracking and Super-Resolution Enhancement
}

\author{
Andrew W. Dowsey ${ }^{1}$, Jennifer Keegan ${ }^{2}$, Mirna Lerotic ${ }^{1}$, Simon Thom ${ }^{3}$, \\ David Firmin $^{2}$, and Guang-Zhong Yang ${ }^{1}$ \\ ${ }^{1}$ Royal Society / Wolfson Foundation Medical Image Computing Laboratory, \\ Department of Computing, Imperial College London, SW7 2BZ, UK \\ \{andrew.dowsey, m.lerotic, g.z.yang\}@imperial.ac.uk \\ ${ }^{2}$ Cardiovascular Magnetic Resonance Unit, National Heart and Lung Institute, \\ Imperial College London, Royal Brompton and Harefield NHS Trust, SW3 6NP, UK \\ j.keegan@rbht.nhs.uk, d.firmin@imperial.ac.uk \\ ${ }^{3}$ International Centre for Circulatory Health, National Heart and Lung Institute, \\ Imperial College London, St. Mary's NHS Trust, W2 1LA, UK \\ s.thomaimperial.ac.uk
}

\begin{abstract}
MR imaging of the heart valve leaflets is a challenging problem due to their large movements throughout the cardiac cycle. This paper presents a motion-compensated imaging approach with COMB tagging for valve imaging. It involves an automatic method for tracking the full 3D motion of the valve plane so as to provide a motion-tracked acquisition scheme. Super-resolution enhancement is then applied to the slice-select direction so that the partial volume effect is minimised. In vivo results have shown that in terms of slice positioning, the method has equivalent accuracy to that of a manual approach whilst being quicker and more consistent. The use of multiple parallel COMB tags will permit adaptive imaging that follows tissue motion. This will have significant implications for quantification of myocardial perfusion and tracking anatomy, functions that are traditionally difficult in MRI.
\end{abstract}

\section{Introduction}

Heart valve disease represents one of the major causes of heart failure. The valves govern the passage of the blood flow within and through the heart. The properties of the valve leaflets are unique; they have high tensile strength, being virtually inextensible at pressures, but are flexible and coordinate forward flow during the cardiac cycle. Understanding valve morphology and interaction with the blood flow could unveil localised factors that contribute to heart diseases. Over the years, different noninvasive 3D imaging techniques have been proposed for assessing valve structure and function. Extensive development in ultrasound imaging has made 3D transthoracic and transesophageal echocardiography an important tool clinically for valve assessment. The alternative of cardiovascular MR offers additional versatility and accuracy in $3 \mathrm{D}$ localisation. This, however, has received limited success mainly due to the difficulty in capturing the moving valve leaflets. 

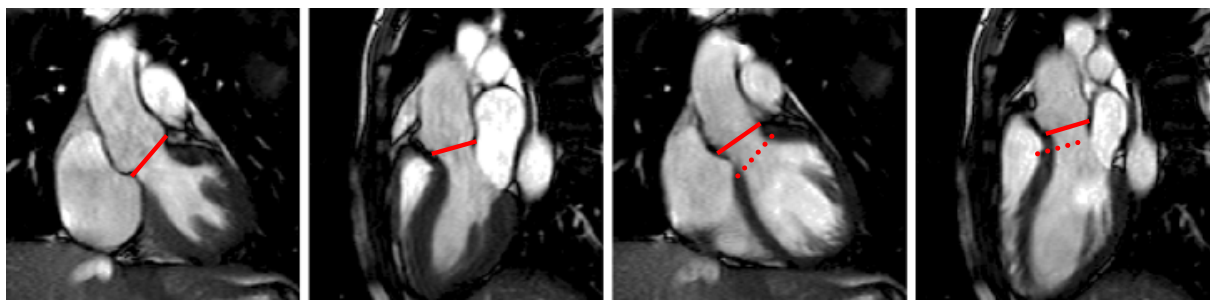

Fig. 1. End systolic (left) and end diastolic (right) frames from 2 orthogonal long axis planes through the aortic valve. The position of othe valve is marked on both frames (solid line). The position of the valve at end systole is superimposed on the end diastolic images (dotted line).

As an example, the aortic valve may be imaged in cross-sections near the base of the left ventricle. However, the basal ventricular plane moves rapidly through the cardiac cycle and so may be displaced by as much as $15-20 \mathrm{~mm}$ between systole and diastole [1], as shown in Fig. 1. This results in the aortic valve moving in and out of the imaging plane, resulting in poor visualisation of the valve leaflets during different phases of the cardiac cycle. To avoid this problem, it is necessary to adaptively track the position of the valve plane during data acquisition so that global cardiac motion is removed to reveal the morphological changes of the valve leaflets. The importance of motion-tracked imaging has been recognised by the MR community for a number of years. Kozerke et al. [2] first introduced tag tracking to perform motion-compensated MR cine velocity mapping through the aortic valve. The basal plane is labelled and then imaged from the four-chamber view plane at regular intervals through the cardiac cycle. The user then marks the septal and left ventricular walls on an arbitrary frame before an automatic technique uses pattern matching to track motion between frames. The technique has been demonstrated useful for quantifying mitral and aortic regurgitation [3]. The disadvantage of this method is that whilst it successfully localises the aortic valve, motion is only tracked on one axis and so it cannot compensate for the complex lateral and torsional motion of the heart.

The purpose of this paper is to develop and validate an automatic scheme to track the full 3D motion of a region of interest (ROI) in the basal ventricular plane to guide the slice position in subsequent motion-tracked acquisition. With this approach, global motion is removed so that the characteristics of the valve leaflets may be clearly observed throughout the cardiac cycle. Super-resolution enhancement is then applied to the slice-select direction so that the partial volume effect is minimised.

\section{Method}

\subsection{COMB Tagging}

The prerequisite of motion-compensated imaging is to tag the target anatomical ROI so that its $3 \mathrm{D}$ position is highlighted. In this study, a cine gradient-echo echo-planar sequence with a tagging pre-pulse [2] was employed. The tagging pre-pulse consists of a selective and non-selective $90^{\circ}$ pulse pair with the selective component involving a COMB excitation [4], which enables the option of simultaneous tagging of multiple 
parallel planes within the same acquisition. The tagged images are generated by the complex subtraction of two datasets, with the phase of the selective component of the tagging pulse pair being reversed between the two. Interleaved vertical long axis (VLA) and horizontal long axis (HLA) image planes were acquired during a single breath-hold, with tagging performed perpendicular to the long axis planes. The resulting images show the motion of the tagged slice through the cardiac cycle in both planes, as illustrated in Fig. 2, such that automated slice tracking can then be applied.
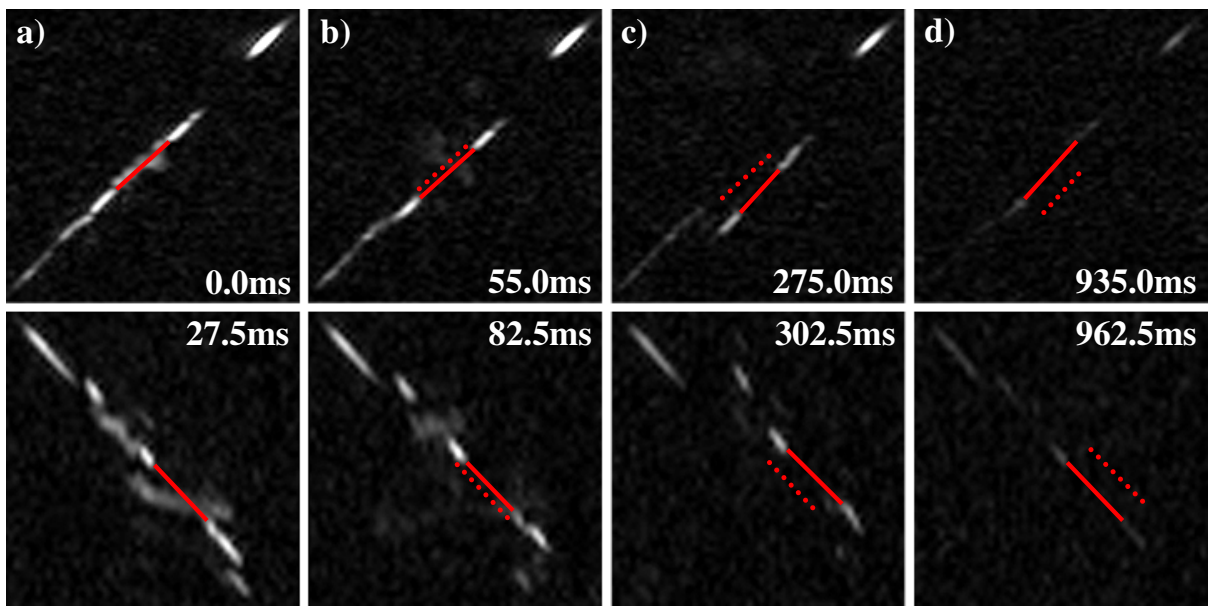

Fig. 2. The basal short axis plane was excited by COMB tagging and VLA (top) and HLA (bottom) acquisitions interleaved through the cardiac cycle so that only motion of the tag plane remains: The left ventricle, which reflects the movement of the aortic valve, is then tracked (solid line). (a) shows tagged blood artefacts; (b) the displacement (dotted line) between successive frames; (c) the position of the tag at maximum displacement; (d) tag fading at end-diastole.

\subsection{Adaptive Motion Tracking}

To determine the required motion-compensation of the valve plane at different phases of the cardiac cycle, multi-resolution image registration of the acquired HLA/VLA image series was performed [5]. Initially, the left ventricle ROI was manually delineated on one chosen VLA and HLA reference frame. The registration process was then performed between neighboring frames, starting with the reference frame and propagating both forwards and backwards. At each stage the initial transformation was computed as the cumulative motion since the reference frame. Due to the removal of all high frequency anatomical information and gradual spatial attenuation of the COMB pre-pulse, the COMB acquisitions provide smooth and continuous derivative data ideal for image registration methods based on quasi-Newtonian optimisation. For this study, the Broyden-Fletcher-Goldfarb-Shanno (BFGS) algorithm [6] was used to optimise a piecewise bilinear mapping to maximise the normalised cross-correlation (NCC) between reference and source images. NCC was employed to compensate for the temporal attenuation of the COMB excitation over time. Performance and convergence was further improved by utilising the closed form partial derivatives of the NCC with respect to the parameters $\Theta$ of the transformation mapping $m$ : 


$$
\begin{gathered}
\frac{\partial}{\partial \Theta} \operatorname{ncc}\left(I_{r}, I_{t}\right)=\frac{\sigma\left(I_{t}\right) \cdot \frac{\partial}{\partial \Theta} \sigma\left(I_{r}, I_{t}\right)-\sigma\left(I_{r}, I_{t}\right) \cdot \frac{\partial}{\partial \Theta} \sigma\left(I_{t}\right)}{\sigma\left(I_{r}\right) \cdot \sigma\left(I_{t}\right)^{2}} \text { where } \\
I_{t}=I_{s} \circ m \quad \frac{\partial}{\partial \Theta} \sigma\left(I_{t}\right)=\frac{\sigma\left(I_{t}, \frac{\partial}{\partial \Theta} I_{t}\right)}{\sigma\left(I_{t}\right)} \quad \frac{\partial}{\partial \Theta} \sigma\left(I_{r}, I_{t}\right)=\sigma\left(I_{r}, \frac{\partial}{\partial \Theta} I_{t}\right) \quad \frac{\partial}{\partial \Theta} I_{t}=\frac{\partial I_{t}}{\partial m} \cdot \frac{\partial m}{\partial \Theta}
\end{gathered}
$$

$I_{r}$ and $I_{s}$ are the reference and source images respectively, $\sigma\left(I_{l}\right)$ represents variance and $\sigma\left(I_{1}, I_{2}\right)$ covariance. To avoid convergence to local maxima a Haar multiresolution pyramid approach was used. The optimisation was performed on Gaussian sub-sampled images from $8 \times 8$ to $128 \times 128$ pixels so that at each stage more and more localised motion was accounted for. This combination of multi-resolution, bilinear mapping and sampling, closed-form derivatives, BFGS optimisation and $\mathrm{C}++$ implementation was capable of motion estimating a 32 frame COMB acquisition (30 registrations) in 16 seconds on a $2 \mathrm{Ghz} \mathrm{PC}$, making it suitable for online processing.

The tracked end-points of the ROI are cubic spline interpolated between timepoints so they can be used to derive the offset and orientation of the tracked slice at any point in the cardiac cycle. It is important to note that due to noise, the points are unlikely to be exactly co-planar, so the orthogonal distance regression plane was obtained. The slice offset (position) vector was defined as the centroid of the ROI endpoints, and the slice orientation (normal) vector was derived using singular value decomposition (SVD) as the eigenvector of $\mathrm{M}^{\mathrm{T}} \mathrm{M}$ corresponding to its smallest eigenvalue, where $\mathrm{M}$ is the matrix of ROI end-points each subtracted by the centroid.

\subsection{Super-Resolution Enhancement}

In 2D multi-slice MRI, the signal to noise ratio (SNR) decreases proportionally with voxel size, since the thinner the slice to be excited by the RF pulse, the sharper the edges of the slice excitation profile and so the longer the pulse duration. In order for the motion-tracked cine acquisition to attain an acceptable SNR, the slice thickness has to be relatively large, which is detrimental to clear depiction of the value leaflets due to a partial volume effect. Super-resolution is a retrospective method to increase resolution and/or increase SNR. This technique reconstructs a high resolution volume from multiple low resolution images with sub-voxel shifts. Due to the band-limited nature of MRI, in-plane resolution cannot be increased retrospectively [7], therefore super-resolution aids only SNR. In this study, super-resolution was performed in the slice-select direction [8]. Overlapping slices were acquired for the motioncompensated acquisition and super-resolved by using a projection onto convex set (POCS) approach [9]. The stack of voxels at each in-plane location were conceptually grouped into $m$ non-overlapping input vectors a, and modelled such that:

$$
\mathbf{a}_{k}=\mathbf{W}_{k} \mathbf{b}+\mathbf{n}_{k}, \text { for } k=1 \ldots m
$$

where $\mathbf{b}$ is the super-resolved output vector to be reconstructed, $\mathbf{W}$ is the transformation matrix mapping each voxel in $\mathbf{b}$ to its constituent voxels in $\mathbf{a}_{1 \ldots \mathrm{m}}$, and $\mathbf{n}$ is noise.

With POCS, a priori knowledge is incorporated via deterministic constraints. Let $\mathbf{C}_{i}=\{\mathbf{u}\}$ be the set of images satisfying constraint $i$ and $\{\hat{\mathbf{u}}\}$ the set that do not. If $\mathbf{C}_{i}$ is closed and convex, the constraint can be defined by a non-expansive projection 
operator $P_{i}$ that maps each $\mathbf{u}$ to itself and each $\hat{\mathbf{u}}$ onto a $\mathbf{u}$ so that $\left|P_{i} \hat{\mathbf{u}}-\hat{\mathbf{u}}\right| \leq|\mathbf{u}-\hat{\mathbf{u}}|$ for all $\mathbf{u}$. POCS then defines the recurrence relation:

$$
\mathbf{u}_{n+1}=P_{n} P_{n-1} \ldots P_{2} P_{1} \mathbf{u}_{n}
$$

which converges weakly from any starting estimate $\mathbf{u}_{\mathbf{0}}$ to a $\mathbf{u}$ in the intersection of all constraints $C_{l} \ldots C_{n}$. Based on the data model in (2), the data consistency constraint set is represented for each pixel $y$ in $\mathbf{y}_{\mathrm{k}}$ as [10]:

$$
C_{k}(y)=\left\{x:\left|r_{x}(y)\right| \leq \delta(y)\right\} \text { where } r_{x}(y)=y-\sum_{x \in \mathbf{x}} x \mathbf{W}_{k}[y ; x]
$$

A benefit of this approach is that $\delta$ represents the statistical confidence we have in the observation, which is set empirically to smooth out aliasing caused by noise and motion artefacts between slices.

\subsection{Data Acquisition and In Vivo Verification}

For in vivo verification, a cine gradient-echo echo-planar sequence with COMB prepulse was implemented on a Siemens Sonata 1.5 Tesla scanner (Erlangen, Germany). The parameters for the imaging sequence were as follows: slice thickness $=12 \mathrm{~mm}$, field of view $=300 \mathrm{~mm} \times 225 \mathrm{~mm}$, matrix size $=128 \times 96$, echo-planar readout factor $=8$, breath-hold duration 20 cardiac cycles, temporal resolution (per interleaved cine pair $=55 \mathrm{~ms}$, starting flip angle $=25^{\circ}$, incrementing to $90^{\circ}$ for the last phase in the cardiac cycle. For the motion-tracked breath-hold acquisitions of the aortic valve, a breath-hold cine segmented FLASH sequence was used. The imaging parameters used were slice thickness $=8 \mathrm{~mm}$, in-plane resolution $=1.2 \mathrm{~mm} \times 2.4 \mathrm{~mm}$, temporal resolution $=50 \mathrm{~ms}$, and breath-hold duration $=16$ cardiac cycles. A total of 20 parallel acquisitions were performed with the slice position offset by $2 \mathrm{~mm}$ on each acquisition ( super-resolution slice overlap $=6 \mathrm{~mm}$ ), resulting in full coverage of the aortic root.

\section{Results}

To demonstrate the effect of slice tracking, Fig. 3 compares a cine acquisition of the aortic valve with and without motion-compensation for one of the 8 subjects studied. In Fig. 3(a), the whole cine was acquired conventionally without slice-tracking. It is evident that cardiac motion has caused the aortic valve to move out of the imaging plane early in the cardiac cycle. In Fig. 3(b), the base of the aortic root was tagged, and its motion through the cardiac cycle determined. The resulting image shows the valve plane within the image slice in all frames of the acquisition, clearly depicting the valve opening/closing during the cardiac cycle. The corresponding POCS superresolution results are shown in Fig. 3(c), demonstrating the increased SNR and reduced partial-volume affect.

To assess the accuracy of the COMB tracking technique, the derived automatic motion-compensation results were compared with manual tracking of the COMB VLA/HLA images. Shapiro-Wilk tests confirmed that residual error between the two approaches was normally distributed. Table 1 summarises the results for the 8 collated COMB tag acquisitions from 5 subjects. The mean, maximum and standard deviation 

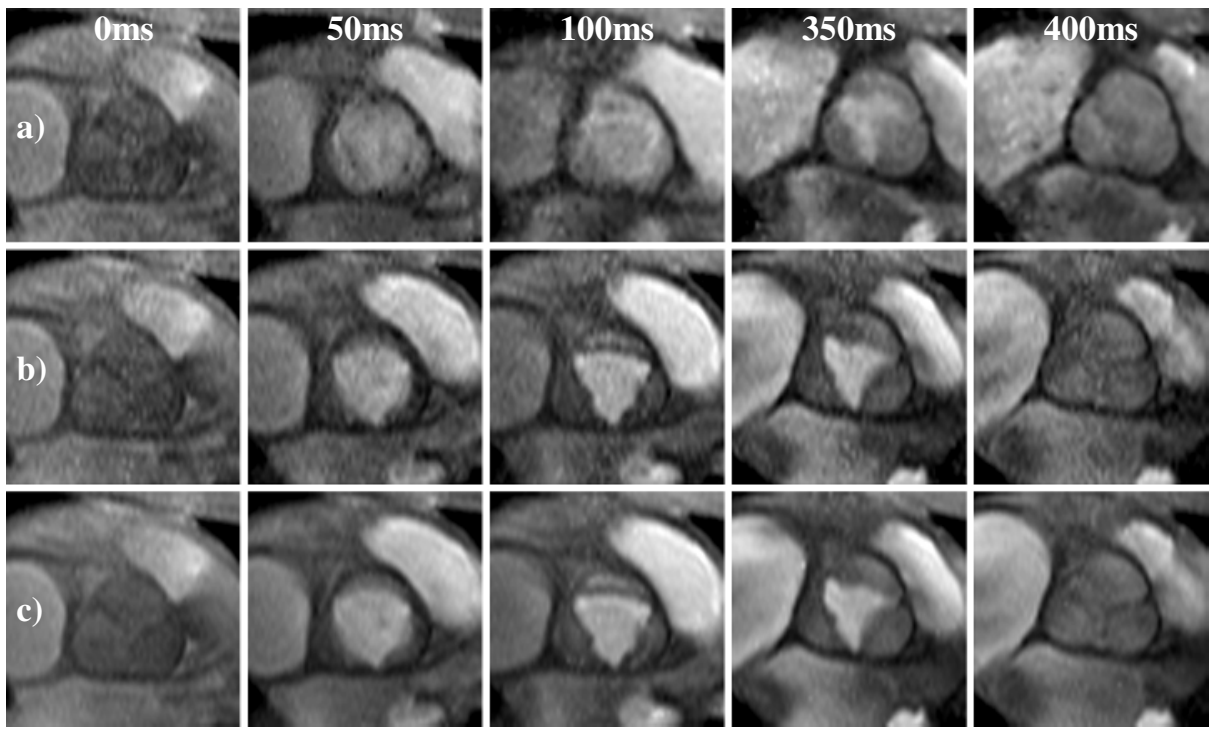

Fig. 3. Cine frames showing the opening and closing of the aortic valve at different phases of the cardiac cycle, where (a) corresponds to image acquisition without motion-tracking, (b) with motion tracking, and (c) motion tracking with super-resolution enhancement.

for the orientation angle and offset distance error between the generated tracked slices for automatic and manual tracking were calculated for each dataset. The manual delineation was highly subjective due to blood artefacts and COMB tag fading (see Fig. 2). In contrast, in the automatic approach only the VLA/HLA pair offering the clearest delineation need be processed manually. Over the 8 datasets the motioncompensation was consistent, only failing on 4 frames due to tag fading. In each case, the mis-tracking could be easily identified and interpolated. The automatic approach with initial ROI delineation and tracking verification took between 20-30 seconds to perform whilst the manual tracking took an average of 3 minutes.

Bland-Altman plots comparing manual $v s$ automatic approaches are presented in Fig. 4(a)(b) for each separate slice parameter in dataset 1 from Table 1. Aortic valve motion varied by $7.3 \mathrm{~mm}$ and $4.8^{\circ}$ in the sagittal axis, $5.4 \mathrm{~mm}$ and $7.7^{\circ}$ in the coronal axis and $12.2 \mathrm{~mm}$ and $7.5^{\circ}$ in the transverse axis, whereas $95 \%$ of residuals were bounded within $1.4 \mathrm{~mm}$ and $2.6^{\circ}$ in the sagittal axis, $1.4 \mathrm{~mm}$ and $3.6^{\circ}$ in the coronal axis and $1.2 \mathrm{~mm}$ and $2.7^{\circ}$ in the transverse axis. In a minority of cases, such as Fig. 4(b)(z), the paired Student's t-test found bias due to the subjective nature of manual ROI delineation. Fig. 4(c) shows the results for a manual vs manual experiment, to gauge the subjective reproducibility. In both manual vs manual and manual vs automatic comparisons, orientation variance was more pronounced than offset variance, though when taken in context of typical aortic valve diameter, an atypical $3^{\circ}$ error would be needed to cause a maximum voxel displacement error of $1 \mathrm{~mm}$. 
Table 1. Statistics of the tracking accuracy for the 8 datasets, illustrating both position and orientation errors between the automatic and manual approaches

\begin{tabular}{c|cc|ccc|ccc}
\hline \multirow{2}{*}{$\begin{array}{c}\text { Data- } \\
\text { set }\end{array}$} & \multirow{2}{*}{$\begin{array}{c}\text { Time- } \\
\text { points }\end{array}$} & $\begin{array}{c}\text { Mis- } \\
\text { tracked }\end{array}$ & \multicolumn{3}{|c|}{ Slice offset error $(\mathrm{mm})$} & \multicolumn{3}{c}{ Orientation error (degrees) } \\
\cline { 4 - 8 } & & mean & sd & max & mean & sd & max \\
\hline 1 & 32 & 0 & 0.55 & 0.22 & 0.97 & 1.22 & 0.71 & 3.23 \\
2 & 24 & 1 & 0.87 & 0.50 & 2.31 & 1.34 & 0.79 & 4.00 \\
3 & 22 & 0 & 0.67 & 0.30 & 1.24 & 1.20 & 0.39 & 1.87 \\
4 & 34 & 2 & 1.29 & 0.91 & 4.03 & 1.46 & 0.67 & 2.77 \\
5 & 22 & 0 & 0.79 & 0.33 & 1.42 & 0.94 & 0.20 & 1.70 \\
6 & 28 & 1 & 1.07 & 0.95 & 4.63 & 1.07 & 0.71 & 3.41 \\
7 & 24 & 0 & 0.80 & 0.33 & 1.46 & 0.88 & 0.25 & 1.88 \\
8 & 24 & 0 & 0.57 & 0.25 & 1.01 & 1.26 & 0.65 & 3.01 \\
\hline
\end{tabular}
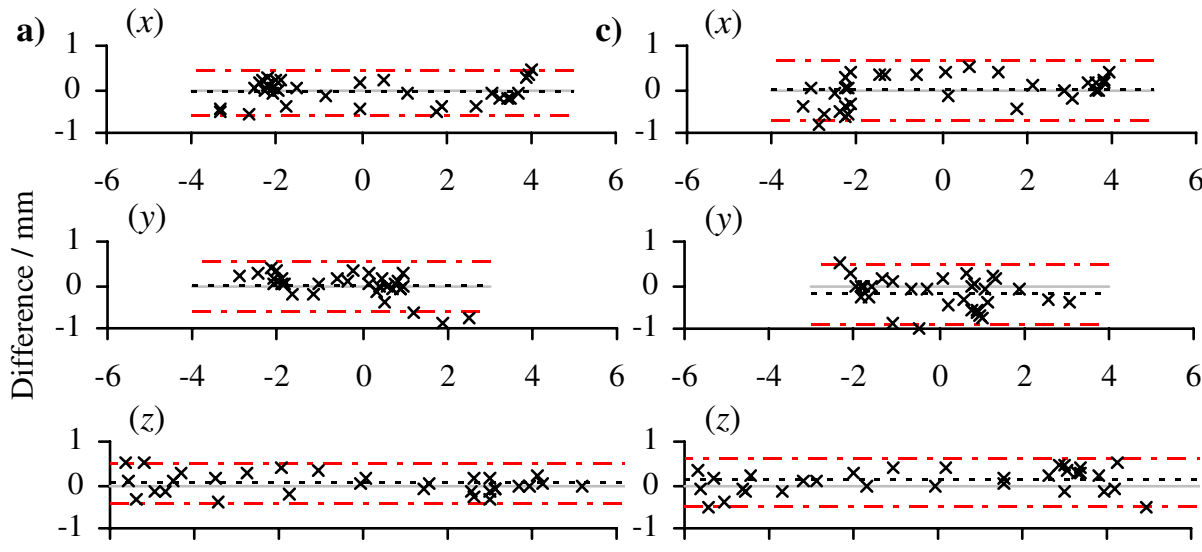

$(z)$

b)

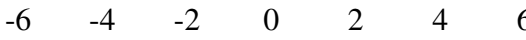

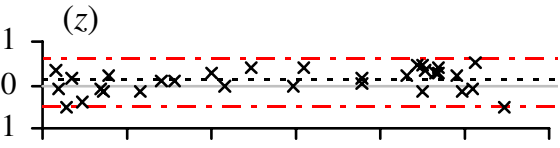

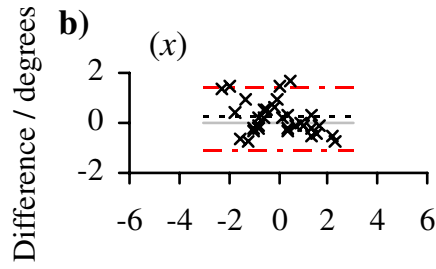

(y)
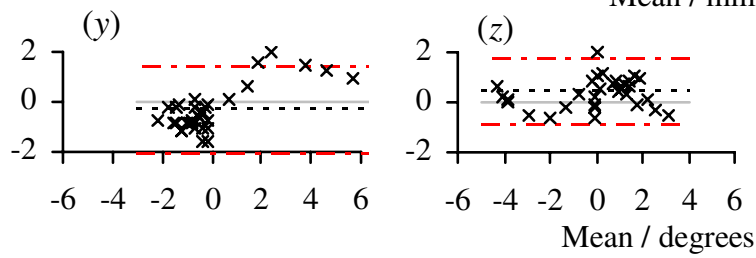

Fig. 4. Bland-Altman plots for dataset 1 in Table 1, comparing the average motion in the sagittal $(x)$, coronal $(y)$ and transverse $(z)$ axes against the difference between: a) automatic and manual tracking of slice offset; b) automatic and manual tracking of slice orientation; c) two manual delineations of slice offset. The mean (dotted line) and two standard deviations either side of the mean (dashed line) are shown. 


\section{Conclusions}

In this study, we have demonstrated that a motion-compensated imaging scheme with COMB tagging provides an effective means of aortic valve imaging. In terms of slice positioning, the method leads to accuracy equivalent to a manual approach but with greater consistency and speed. In this study, the slice-tracked valve images were acquired with a segmented FLASH imaging sequence which can easily be modified to allow phase velocity mapping and thereby slice-followed flow measurements throughout the cardiac cycle. The use of multiple parallel COMB tags will permit adaptive imaging that follows tissue motion. This will have significant implications for improved tracking of cardiac motion, a challenge that has hitherto been difficult with MRI. Such tracking applied to the coronary arteries will facilitate investigation of the progression of atherosclerosis.

Acknowledgement. This work was supported by British Heart Foundation grant PG/04/078/17370.

\section{References}

1. Karwatowski, S.P., Mohiaddin, R., Yang, G.-Z. Firmin, D.N, Sutton, M.S., Underwood, S.R., Longmore, D.B.: Assessment of regional left ventricular long-axis motion with MR velocity mapping in healthy subjects. J. Magn. Reson. Imaging 4 (1994) 151-155

2. Kozerke, S., Scheidegger, M. B., Pedersen, E. M., Boesiger, P.: Heart motion adapted cine phase-contrast flow measurements through the aortic valve. Magn. Reson. Med. 42 (1999) 970-978

3. Kozerke, S., Schwitter, J., Pedersen, E.M., Boesiger, P.: Aortic and mitral regurgitation: Quantification using moving slice velocity mapping. J. Magn. Reson. Imaging 14 (2001) 106-112

4. Dumoulin, C.L., Doorly, D.J., Caro, C.G.: Quantitative measurement of velocity at multiple positions using COMB excitation and fourier velocity encoding. Magn. Reson. Med. 29 (1993) 44-52

5. Veeser, S., Dunn, M.J., Yang G.-Z.: Multiresolution image registration for twodimensional gel electrophoresis. Proteomics 1 (2001) 856-870

6. Nocedal, J.: Updating quasi-Newton matrices with limited storage. Math. Comp. 35 (1980) 773-782

7. Greenspan, H., Oz, G., Kiryati, N., Peled, S.: MRI inter-slice reconstruction using superresolution. Magn. Reson. Imaging 20 (2002) 437-446

8. Peeters, R.R., Kornprobst, P., Nikolova, M., Sunaert, S., Vieville, T., Malandain, G., Deriche, R., Faugeras, O., Ng, M., Van Hecke, P.: The use of super-resolution techniques to reduce slice thickness in functional MRI. Int. J. Imaging Sys. Tech. 14 (2004) 131-138

9. Stark, H., Oskoui, P.: High-resolution image recovery from image-plane arrays, using convex projections. J. Opt. Soc. Amer. A 11 (1989) 1715-1726

10. Tekalp, A. M., Ozkan, M. K., Sezan, M. I.: High-resolution image reconstruction from lower-resolution image sequences and space-varying image restoration. Proc. IEEE ICASSP 3 (1992) 167-172 